\title{
Simulação estocástica da radiação fotossinteticamente ativa e da temperatura do ar por diferentes métodos
}

\author{
Thomas Newton Martin(1), Lindolfo Storck ${ }^{(2)}$ e Durval Dourado Neto(3)
}

\begin{abstract}
(1)Universidade Tecnológica Federal do Paraná, Estrada para Boa Esperança, Km 4, Caixa Postal 157, CEP 85660-000 Dois Vizinhos, PR. E-mail: thomas.martin@hotmail.com (2)Universidade Federal de Santa Maria, Centro de Ciências Rurais, Dep. de Fitotecnia, Camobí, CEP $97105-900$ Santa Maria, RS. E-mail: lindolfo@smail.ufsm.br ${ }^{(3)}$ Escola Superior de Agricultura Luiz de Queiroz, Dep. de Produção Vegetal, Caixa Postal 9 , CEP 13418-900 Piracicaba, SP. E-mail: dourado@esalq.usp.br
\end{abstract}

Resumo - O objetivo deste trabalho foi comparar três métodos para simulação de dados de radiação fotossinteticamente ativa e temperatura do ar, com o uso de parâmetros diários de 17 estações do Estado de São Paulo. A simulação foi realizada para o primeiro e o $16^{\circ}$ dia de cada mês, conforme: a distribuição normal truncada a mais ou menos 1,96 de desvio-padrão; distribuição triangular assimétrica; e distribuição normal bivariada. As estimativas com os dados simulados foram comparadas com os respectivos parâmetros (obtidos com os dados observados), pelos testes de homogeneidade de variância F e Bartlett, teste t de comparação de médias, coeficiente de correlação de Pearson, índice de concordância de Willmott, índice de desempenho de Camargo, coeficiente angular e o teste de normalidade dos dados. A simulação pela distribuição normal bivariada é a mais adequada para representar as variáveis climáticas.

Termos para indexação: distribuições de probabilidade, variáveis climáticas, normal bivariada, distribuição normal, distribuição triangular.

\section{Random simulation of photosynthetically active radiation and air temperature through different methods}

\begin{abstract}
The purpose of this work was to compare three methods for simulating data of photosynthetically active radiation and air temperature, using daily parameters of 17 stations of São Paulo State, Brazil. The simulation of those elements was carried out for de $1^{\text {st }}$ and the $16^{\text {th }}$ day of each month, through three cases: nonsymmetric triangular distribution; normal distribution truncated at 1.96 standard deviation; and bivaried normal distribution. The simulated data were evaluated through the tests of homogeneity of variance $\mathrm{F}$ and Bartlett, $\mathrm{t}$ test, agreement index of Willmott, angular coefficient of the straight line, the index of performance of Camargo and tack the normal distribution (uni-varied). The simulation using the bi-varied normal distribution is most appropriate for representing the climate variables.

Index terms: probability distributions, climatic variables, bivariate normal, normal distribution, triangular distribution.
\end{abstract}

\section{Introdução}

A utilização de técnicas de modelagem permite a criação de cenários, pelas simulações sobre um conjunto de equações que irão representar um processo. No caso da cultura da soja, podem-se estimar rendimentos de grãos potenciais e penalizados. A partir daí, pode-se traçar um conjunto de medidas que objetivam a obtenção desses rendimentos e, conseqüentemente, auxiliar as instituições públicas e privadas para uma tomada de decisão correta. Vantagens como a economia de tempo, trabalho e quantidade de recursos, de ordem financeira e logística, serão obtidas pelo uso de modelos de culturas (Pessoa et al., 1997). Assim, estudos na área de modelagem auxiliarão no planejamento e tomada de decisões de manejo e estratégias de gerenciamento do setor agrícola. A utilização de modelos de culturas, em associação a técnicas de geoprocessamento, facilitará a identificação de locais e épocas mais recomendadas para o desenvolvimento de determinada cultura, tornando-se uma importante ferramenta para o zoneamento agrícola e difusão de informações, como utilizado por Carvalho Junior et al. (2003) no zoneamento agropedoclimático da cultura da soja no Estado do Rio Grande do Sul. 
A estimação de rendimentos potenciais para determinada região está na dependência do conhecimento de séries históricas de variáveis climáticas como radiação solar global, temperatura, precipitação, umidade relativa do ar, entre outras. Porém, com a utilização de séries históricas reduzidas existe maior probabilidade de os resultados serem tendenciosos (Genneville \& Boock, 1983). A utilização de recursos computacionais para a simulação estocástica permite a geração de valores diários e imprime maior confiabilidade dos resultados obtidos (Sousa, 1999).

A mudança do estádio fenológico, para algumas culturas, depende essencialmente da temperatura, como no caso do milho, pelo acúmulo de unidades térmicas acima da temperatura mínima para o crescimento. Esse também é definido como graus-dia, que é a diferença entre a temperatura média diária e a temperatura mínima ou temperatura base exigida por uma espécie (Villa Nova et al., 1972). No caso da cultura da soja, elevadas temperaturas do ar durante o crescimento reduzem o tempo para o florescimento (Major et al., 1975). Em condições tropicais e subtropicais, baixas temperaturas limitam severamente o crescimento das plantas, cuja capacidade fotossintética é reduzida em razão do decréscimo da eficiência quântica no fotossistema II e atividades do fotossistema I, e ocorre também redução dos ciclos para a formação de enzimas e ATP, no estroma das plantas C3 (Sonoike, 1998; Allen \& Ort, 2001).

A partir da radiação fotossinteticamente ativa e a temperatura média do ar, é possível estimar a máxima assimilação de dióxido de carbono para determinada cultura (Van Heemst, 1986). Por meio de tal método, foi possível a estimação do rendimento produtividade potencial para as culturas do milho (Dourado Neto et al., 2004) e das cultivares Marandu e Xaraés da Brachiaria brizantha (Detomini \& Dourado Neto, 2005).

A técnica de geração de pares de valores (radiação solar e temperatura média do ar), com distribuição normal truncada, foi aplicada para a cultura do milho (Dourado Neto et al., 2005; Assis et al., 2006). Neste método, os valores de radiação e temperatura são simulados independentemente, ao simular uma enquanto fixa a outra e vice-versa. Além disso, cada valor é simulado pela distribuição normal, em que valores simulados menores que a "média menos 1,96 desvio-padrão" ou maiores que a "média mais 1,96 desvio-padrão" são descartados (truncada).
A distribuição de probabilidade triangular é utilizada nos casos em que é possível determinar o valor mais provável da variável aleatória, os valores mínimos e máximos, e quando uma função linear parece apropriada para a descrição da distribuição dos valores dos erros das variáveis. Nesses casos, pode-se utilizar a distribuição triangular, que é um modelo adequado entre as distribuições normal e uniforme. A distribuição triangular foi aplicada por Detomini \& Dourado Neto (2005) e Assis et al. (2006), para a simulação de valores médios de temperatura e radiação solar global, que foram utilizados para estimar o rendimento potencial e penalizado de cultura de milho e Brachiaria.

É necessário melhorar a representação dos valores diários da interação radiação solar global (radiação fotossinteticamente ativa) e temperatura do ar, pois nos trabalhos de simulação produzidos atualmente, a radiação fotossinteticamente ativa e a temperatura do ar são simuladas independentemente, apesar de serem relacionadas, como realizado por Dourado Neto et al. (2005) e Assis et al. (2006). Existe, portanto, uma lacuna na literatura especializada que possa indicar uma forma de simulação que considere a relação entre as variáveis (radiação e temperatura).

O objetivo deste trabalho foi comparar três métodos para a simulação da radiação fotossinteticamente ativa e da temperatura média do ar, usandos os dados diários de 17 estações climatológicas do Estado de São Paulo.

\section{Material e Métodos}

Os dados referentes às variáveis meteorológicas insolação e temperatura média do ar, de 17 locais do Estado de São Paulo, foram obtidos no Departamento de Ciências Exatas da Esalq, Piracicaba (1926-2005); e no Instituto Agronômico (IAC), relativos às seguintes cidades: Campinas (1960-2004), Cravinhos (1997-2002), Capão Bonito (1991-2002), Gália (1977-2002), Itararé (1977-2000), Jaú (1955-2002), Jundiaí (1993-1999), Limeira (1980-2004), Mococa (1959-2000), Monte Alegre do Sul (1981-2000), Pariqüeraçu (1980-2000), Pindamonhangaba (1982-2000), Pindorama (1965-2000), Ribeirão Preto (1965-2000), Tatuí (1992-2000) e Tietê (1991-2000). Primeiramente, foram estimados os valores de radiação fotossinteticamente ativa, a partir dos valores de insolação, conforme Pereira et al. (2002).

O banco de dados foi formado por 1.000 valores simulados das variáveis, em cada uma das 24 datas propostas (1ํ e 16을 dias de cada mês). Os dados foram 
simulados segundo: a distribuição normal truncada a mais ou menos 1,96 desvio-padrão; distribuição triangular assimétrica; e distribuição normal bivariada. Nas duas primeiras situações, a simulação dos valores de temperatura e de radiação fotossinteticamente ativa foi realizada independentemente, tendo-se desconsiderado a relação de dependência entre as variáveis. Na terceira situação de simulação, estimou-se, primeiramente, a radiação fotossinteticamente ativa e, a partir dessa, simulou-se a temperatura média do ar (tendo-se considerado a relação de dependência).

A função de distribuição de probabilidade normal truncada, usada na simulação, é definida por (Kortum, 2002):

$$
f(x)=\left\{\begin{array}{cc}
0, & x \leq a \\
\frac{1}{\sqrt{2 \pi \sigma^{2}}} \exp \left\{-\frac{1}{2}\left(\frac{x-\mu}{\sigma}\right)^{2}\right\}, & a<x<b \\
0, & x \geq b
\end{array}\right.
$$

em que $\mu$ e $\sigma$ representam a média e o desvio-padrão da distribuição normal (não truncada); $a=-1,96$; $\mathrm{b}=1,96$.

A função densidade de probabilidade triangular, usada na simulação, é definida por:

$$
f(x)=\left\{\begin{array}{cl}
\frac{2(x-a)}{(m-a)(b-a)}, & a \leq X \leq m \\
\frac{2(b-x)}{(b-m)(b-a)} & , m \leq X \leq b \\
0 & , X<a \text { ou } X>b
\end{array}\right.
$$

em que: $\mathrm{m}$ é o valor mais provável; a é o valor mínimo; e b é o valor máximo da variável. Essa metodologia de simulação de dados pela distribuição triangular foi aplicada por Detomini \& Dourado Neto (2005) e Assis et al. (2006).

Para a simulação de pares de valores, com distribuição normal bivariada, estimaram-se, inicialmente, duas variáveis aleatórias com distribuições uniformes, no intervalo 0 e 1 , e independentes $\left(\mathrm{u}_{1}\right.$ e $\left.\mathrm{u}_{2}\right)$. Em seguida, cada uma dessas variáveis foi transformada para distribuição normal padrão $(\mu=0$ e $\sigma=1)$, segundo Box \& Muller (1958), ou seja:

$\mathrm{x}_{1}=\left[\left(-2 \ln \left(\mathrm{u}_{1}\right)\right]^{0,5} \cos \left(2 \pi \mathrm{u}_{2}\right) \mathrm{e}_{2}=\left[\left(-2 \ln \left(\mathrm{u}_{1}\right)\right]^{0,5} \operatorname{sen}\left(2 \pi \mathrm{u}_{2}\right)\right.\right.$, com $\mathrm{x}_{1}$ independente de $\mathrm{x}_{2}$. Devem-se utilizar as funções seno e co-seno em unidades de radianos. Cada par de valores da radiação fotossinteticamente ativa $\left(R_{i}\right)$ e da temperatura do $\operatorname{ar}\left(\mathrm{T}_{\mathrm{i}}\right)$, com adaptações de Hogg \& Craig (1978), foi obtida por:

$\mathrm{R}_{\mathrm{i}}=\mu_{\mathrm{R}}+\sigma_{\mathrm{R}} \mathrm{X}_{1} \mathrm{e} \mathrm{T}_{\mathrm{i}}=\left[\mu_{\mathrm{T}}+\left(\rho \sigma_{\mathrm{T}} / \sigma_{\mathrm{R}}\right)\left(\mathrm{R}_{\mathrm{i}}-\mu_{\mathrm{R}}\right)\right]+\left(1-\rho^{2}\right)^{0,5} \sigma_{\mathrm{T}} \mathrm{X}_{2}$, em que $\mu_{\mathrm{T}}$ e $\sigma_{\mathrm{T}}$ são, respectivamente, a média e o desviopadrão paramétrico da temperatura do ar $(\mathrm{T})$ de um dado local e dia; $\mu_{R}$ e $\sigma_{R}$ são, respectivamente, a média e o desvio-padrão paramétrico da radiação fotossinteticamente ativa (R) de um dado local e dia; e $\rho$ é a correlação paramétrica entre $\mathrm{T}$ e $\mathrm{R}$ para um dado local e dia.

Os processos de simulação da temperatura e da radiação fotossinteticamente ativa foram avaliados por comparação dos parâmetros (média, variância e correlação) dos dados originais, com os parâmetros das 1.000 simulações realizadas, para cada local, em todos os dias do ano. A média estimada pelo processo simulado - normal truncado, triangular e normal bivariado - foi comparada com a média paramétrica da respectiva variável (radiação e temperatura), pelo teste $\mathrm{t}$ bilateral a 5\% de probabilidade de erro. As variâncias foram comparadas pelo teste $\mathrm{F}$, razão da variância simulada com a paramétrica, a 5\% de probabilidade de erro (Steel et al., 1997). A técnica de regressão simples, cuja reta passa pela origem do sistema de eixos coordenados foi também utilizada (Morettin \& Bussab, 2003).

Para validar os processos de simulação, utilizaramse, complementarmente, indicadores estatísticos como: o coeficiente de correlação de Pearson (r), entre os valores observados e os valores simulados por cada um dos processos (Morettin \& Bussab, 2003); o índice de concordância de Willmott (Id), dado por uma aproximação matemática que avalia a exatidão e avalia o afastamento dos valores simulados em relação aos observados (Willmott, 1981), onde a variação é de zero (nenhuma concordância) até a unidade (concordância perfeita); e o índice de desempenho de Camargo ( $C=r^{*}$ Id), que se constitui no produto dos dois índices anteriores (Camargo \& Sentelhas, 1997). Foram, também, aplicados os testes de normalidade (Kolmogorow-Smirnov) (Campos, 1983) aos valores simulados, tendo-se usado a média e a variância dos valores observados como parâmetros da distribuição normal, e o teste de Bartlett (Steel et al., 1997) para verificar a homogeneidade das variâncias dos valores simulados em relação aos observados. Com tais procedimentos, poder-se-á identificar o processo de 
simulação que mais reproduz os dados observados, para qualquer local e dia do ano.

\section{Resultados e Discussão}

Na Tabela 1, estão descritos os valores originais de média, desvio-padrão, covariância e correlação para radiação fotossinteticamente ativa e temperatura média do ar (média para 17 locais), nas 24 datas do ano. Esses resultados foram os usados para comparar os obtidos para a simulação dos dados pelos três métodos. Apesar de os valores de correlação apresentados na Tabela 1 variarem de $-0,16$ a 0,58 , ao se analisarem os valores durante o ano em cada local (e não na média), verificase que a correlação pode variar de $-0,30$ a 0,75 . Esses valores de correlação são importantes como parâmetros, para a simulação de dados por meio da distribuição normal bivariada. Então, verifica-se que na natureza (dados observados) existe uma correlação entre as duas variáveis, que é positiva durante os meses quentes e negativa ou baixa durante os meses de inverno. Esses últimos caracterizam um período seco, com menor incidência de nuvens e, por isso, com maior radiação fotossinteticamente ativa disponível e a temperatura mais baixa devido à inclinação da terra em relação à radiação incidente.

Os resultados das estatísticas, com a simulação baseada na distribuição triangular assimétrica, permitem inferir que apesar de existir uma relação de dependência entre a radiação fotossinteticamente ativa $(R)$ e a temperatura $(\mathrm{T})$ na natureza, nessa modalidade de simulação, os resultados entre as duas variáveis (R e T) apresentou correlação bem próxima à nulidade em todas as 24 datas (Tabela 1). Certamente, isso ocorre em razão do processo de simulação de $\mathrm{R}$ independente de $\mathrm{T}$.

Para a simulação normal truncada a $\pm 1,96$ desviopadrão, se verifica, pelas estatísticas, que a simulação dos dados não proporciona uma correlação entre $\mathrm{R}$ e T, fato que também pode ser atribuído ao processo de simulação de R independente de $\mathrm{T}$ (Tabela 2). Ao se

Tabela 1. Média $(\mu)$, desvio-padrão $(\sigma)$, covariância (Cov) e correlação $(\rho)$, entre os valores da radiação fotossinteticamente ativa (RFA) e temperatura do ar (Temp), observados em 24 datas e 17 locais ${ }^{(1)}$ do Estado de São Paulo ${ }^{(2)}$.

\begin{tabular}{|c|c|c|c|c|c|c|c|c|c|c|c|c|c|c|c|c|c|c|}
\hline \multirow[t]{3}{*}{ Datas } & \multicolumn{6}{|c|}{ Valores observados (parâmetros) } & \multicolumn{12}{|c|}{ Valores simulados (triangular assimétrica) } \\
\hline & \multicolumn{2}{|c|}{ RFA } & \multicolumn{2}{|c|}{ Temp } & \multirow[t]{2}{*}{$\operatorname{Cov}$} & \multirow[t]{2}{*}{$\rho$} & \multicolumn{4}{|c|}{ RFA } & \multicolumn{4}{|c|}{ Temp } & \multirow[t]{2}{*}{$\mathrm{Cov}$} & \multirow[t]{2}{*}{ DIF } & \multirow[t]{2}{*}{$\rho$} & \multirow[t]{2}{*}{ DIF } \\
\hline & $\mu$ & $\sigma$ & $\mu$ & $\sigma$ & & & $\hat{\mu}$ & DIF & $\hat{\sigma}$ & DIF & $\hat{\mu}$ & DIF & $\hat{\sigma}$ & DIF & & & & \\
\hline 1/jan. & 215,99 & 5,71 & 4,16 & 2,05 & 37,59 & 0,37 & 16,61 & 0,63 & 32,65 & 23,06 & 23,32 & 0,84 & 1,75 & 0,30 & 0,85 & 30,13 & 0,01 & 0,36 \\
\hline 16/jan. & & 54,65 & 24,55 & 2,08 & 34,81 & 0,40 & & 1,04 & 68 & 22,97 & 4,44 & 0,11 & 1,73 & 0,35 & 0,10 & 4,71 & 0,00 & 0,40 \\
\hline $1 /$ fev. & 12,95 & 48,19 & 24,63 & 2,13 & 37,66 & 0,49 & 209,50 & 3,45 & 29,74 & 18,45 & 24,38 & 0,25 & 1,69 & 0,44 & $-0,08$ & 37,74 & 0,00 & 0,49 \\
\hline $16 /$ fev. & 213,54 & 51,53 & 24,41 & 1,79 & 40,43 & 0,58 & 10,21 & 3,33 & 31,13 & 20,40 & 24,24 & 0,17 & 1,40 & 0,39 & $-0,24$ & 40,67 & 0,00 & 0,58 \\
\hline $1 /$ mar. & 200,52 & 48,92 & 24,15 & 1,82 & 31,87 & 0,38 & 199,51 & 1,01 & 28,82 & 20,10 & 23,70 & 0,44 & 1,48 & 0,34 & 0,21 & 31,66 & 0,00 & 0,37 \\
\hline $16 /$ mar. & 206,90 & 41,05 & & & 6,75 & & & 7,35 & & 14,31 & 3,46 & 01 & 1,36 & 0,53 & 0,25 & 6,50 & & 0,11 \\
\hline 1/abr. & 7,85 & 40,66 & 24 & 1,77 & 19,22 & 0,28 & 80 & 6,05 & 26,09 & 14,57 & 22,92 & 0,32 & 1,31 & 0,46 & $-0,25$ & 19,47 & $-0,01$ & 0,29 \\
\hline 16/abr. & 1,53 & 38,39 & 22,05 & 2,61 & 1,16 & 0,05 & 174,28 & 7,25 & 24,51 & 13,88 & 21,22 & 0,83 & 1,95 & 0,65 & $-0,01$ & 1,17 & 0,00 & 0,05 \\
\hline $1 /$ maio &, 39 & 32,83 & 10 & 2,61 & 6,86 & 0,11 & & 8,70 & 22,50 & 10,33 &, 73 & 0,37 & 2,13 & 0,48 & 0,68 & 6,18 & 0,02 & 0,10 \\
\hline $16 /$ maio & 49,66 & 37,19 & 19,28 & 2,46 & 9,14 & 0,12 & 6,03 & 3,63 & 22,38 & 14,82 & 18,89 & 0,38 & 1,82 & 0,64 & 0,36 & 8,77 & 0,01 & 0,11 \\
\hline & 148,75 & 33,30 & 17,22 & 2,99 & $-11,94$ & $-0,13$ & 140,91 & 7,83 & 21,28 & 12,02 & 16,51 & 0,72 & 2,34 & 0,65 & $-0,48$ & 46 & & 12 \\
\hline 16/jun. & 145,82 & 28,29 & 17,57 & 2,45 & 13,35 & 0,21 & 39,89 & 5,94 & 40 & 9,89 & 17,39 & 0,18 & 1,77 & 0,69 & 0,00 & 13,35 & 0,00 & 0,21 \\
\hline 1/jul. & & 24,83 & 18,11 & & 1,67 & & & & & 7,54 & 25 & 35 & 2,12 & 0,30 & 0,20 & 1,47 & 0,00 & 0,04 \\
\hline 16/jul. & 152,88 & 30,94 & 63 & 3,03 & $-1,64$ & $-0,06$ & 88 & 7,01 & 20,34 & 10,60 & 17,51 & 0,12 & 2,30 & 0,73 & $-0,25$ & 1,39 & 0,00 & 0,07 \\
\hline 1/ago. & 170,85 & 27,60 & 18,13 & 3,08 & 20,83 & & 159,55 & 11,30 & 20,25 & 7,35 & 16,96 & 1,17 & 2,60 & 0,47 & 0,42 & 20,41 & 0,00 & 0,35 \\
\hline 16/ago. & 182,70 & 31,07 & 19,23 & 3,21 & $-11,99$ & $-0,16$ & 170,53 & 12,17 & 23,03 & 8,04 & 18,42 & 0,81 & 2,60 & 0,61 & 0,45 & 12,44 & 0,00 & 0,16 \\
\hline $1 /$ set. & 183,77 & 39,78 & 20,38 & & 27,18 & & 179,47 & & 24,89 & 14,89 & 20,13 & 0,25 & 2,26 & 0,74 & $-0,64$ & 27,83 & 0,00 & 0,29 \\
\hline & & & & & & & & & & & & 39 & 2,26 & 0,69 & & 6,26 & & 0,02 \\
\hline 1/out. & 198,59 & 49,54 & 20,99 & 2,39 & 9,48 & 0,08 & 196,33 & 2,26 & 29,02 & 20,52 & 20,91 & 0,08 & 1,97 & 0,41 & $-0,59$ & 10,07 & 0,00 & 0,07 \\
\hline 16/out. & 203,99 & 56,96 & 22,40 & 2,84 & 10,96 & 0,02 & 201,97 & 2,02 & 31,93 & 25,03 & 22,44 & 0,04 & 2,08 & 0,76 & $-0,26$ & 11,22 & 0,00 & 0,01 \\
\hline $1 /$ nov. & 216,64 & 51,69 & 22,63 & 2,99 & 10,43 & 0,06 & 211,86 & 4,78 & 31,96 & 19,73 & 22,43 & 0,20 & 2,18 & 0,81 & $-0,22$ & 10,65 & 0,00 & 0,05 \\
\hline & 239,69 & 50,81 & 23,16 & 2,65 & 17,59 & & 230,47 & 9,23 & 32,40 & 18,41 & 22,91 & 0,25 & 2,15 & 0,50 & $-1,01$ & 18,61 & 0,00 & 0,18 \\
\hline 1/dez. & 220,10 & 57,36 & 23,39 & 2,10 & 43,46 & 0,47 & 218,95 & 1,15 & 32,92 & 24,44 & 23,12 & 0,26 & 1,61 & 0,49 & $-0,43$ & 43,89 & 0,00 & 0,46 \\
\hline 16/dez. & 222,78 & 53,35 & 23,88 & 2,07 & 21,48 & 0,27 & 219,54 & 3,24 & 32,92 & 20,42 & 23,24 & 0,63 & 1,65 & 0,42 & 0,34 & 21,14 & 0,00 & 0,26 \\
\hline Média & & & & & & & & 5,20 & & 16,15 & & 0,40 & & 0,54 & & 18,07 & & 0,21 \\
\hline
\end{tabular}

(1)Campinas (1960-2004), Cravinhos (1997-2002), Capão Bonito (1991-2002), Gália (1977-2002), Itararé (1977-2000), Jaú (1955-2002), Jundiaí (1993-1999), Limeira (1980-2004), Mococa (1959-2000), Monte Alegre do Sul (1981-2000), Pariqüeraçu (1980-2000), Pindamonhangaba (1982-2000), Pindorama (1965-2000), Piracicaba (1926-2005), Ribeirão Preto (1965-2000), Tatuí (1992-2000) e Tietê (1991-2000). ${ }^{(2)}$ As estimativas de $\mu$ e $\sigma$ dos valores de RFA e Temp foram simuladas pela distribuição triangular, diferença absoluta (DIF) entre os valores observados e as respectivas estatísticas dos dados simulados. 
analisarem as estatísticas para os dados simulados, pela distribuição normal bivariada, observa-se que a relação de dependência entre $\mathrm{R}$ e $\mathrm{T}$, médias e desvio-padrão, proporcionam valores mais fidedignos aos parâmetros estabelecidos.

Pode ser observado, pela média dos valores absolutos das diferenças entre os parâmetros e os valores simulados, que em praticamente em todas as situações, comportou-se de maneira inferior, ou bastante similar, quando comparados com a simulação triangular assimétrica e normal truncada. Além disso, a situação apresentada pela simulação normal bivariada faz com que os valores sejam mais próximos aos parâmetros, em razão da correlação que existe entre as duas variáveis.

O comportamento das variáveis simuladas se verifica pela correlação entre os parâmetros da radiação fotossinteticamente ativa e as estatísticas dos valores simulados da mesma radiação fotossinteticamente ativa para o determinado dia juliano (Tabela 3). Além disso, são apresentados os índices de concordância de Willmott, o coeficiente angular da reta e o índice de desempenho de Camargo, para os três processos de simulação.

A correlação entre os parâmetros e as estatísticas dos dados simulados é elevada nos processos de simulação normal truncada e normal bivariada. Em contrapartida, a simulação triangular deixa a desejar, como se deduz ao se interpretar o índice de concordância de Willmott, coeficiente angular da reta e índice de desempenho de Camargo. É conveniente que a escolha de determinado processo de simulação dos dados considere o maior número de testes estatísticos ou critérios possíveis, para se realizar a escolha adequada.

Dourado Neto et al. (2005) analisaram dois casos, para caracterizar a distribuição temporal (valores diários) das séries históricas de temperatura e de radiação solar global. No primeiro caso, os autores utilizaram a distribuição normal truncada a $\pm 1,96$ desvio-padrão, com a temperatura variável e a radiação solar global diária constante. No segundo caso, utilizaram a mesma distribuição, porém a radiação solar global era variável

Tabela 2. Estimativas da média $(\mu)$ e do desvio-padrão $(\sigma)$, dos valores da radiação fotossinteticamente ativa (RFA) e temperatura do ar (Temp), observados em 24 datas e 17 locais $^{(1)}$ do Estado de São Paulo, simulados pela distribuição normal truncada e normal bivariada, diferenças absolutas (DIF) com os respectivos valores observados (Tabela 1), covariância (Cov) e correlação ( $\rho)$ entre os valores simulados e observados com respectivas diferenças.

\begin{tabular}{|c|c|c|c|c|c|c|c|c|c|c|c|c|c|c|c|c|c|c|c|c|c|c|c|c|}
\hline \multirow[t]{3}{*}{ Datas } & \multicolumn{12}{|c|}{ Valores simulados (normal truncada) } & \multicolumn{12}{|c|}{ Valores simulados (normal bivariada) } \\
\hline & \multicolumn{4}{|c|}{ RFA } & \multicolumn{4}{|c|}{ Temp } & \multirow[t]{2}{*}{ Cov } & \multirow[t]{2}{*}{ DIF } & \multirow[t]{2}{*}{$\rho$} & \multirow[t]{2}{*}{ DIF } & \multicolumn{4}{|c|}{ RFA } & \multicolumn{4}{|c|}{ Temp } & \multirow[t]{2}{*}{$\mathrm{Cov}$} & \multirow[t]{2}{*}{ DIF } & & \multirow[t]{2}{*}{ DIF } \\
\hline & $\hat{\mu}$ & DIF & $\hat{\sigma}$ & DIF & $\hat{\mu}$ & DIF & $\hat{\sigma}$ & DIF & & & & & $\hat{\mu}$ & D IF & $\hat{\sigma}$ & $\mathrm{DIF}$ & $\hat{\mu}$ & DIF & $\hat{\sigma}$ & DIF & & & & \\
\hline 1/jan. & 216,25 & 0,26 & 48,57 & 7,14 & 23,97 & 0,19 & 1,64 & 0,42 & 0,30 & 37,29 & 0,00 & 0,37 & 215,88 & 0,11 & 55,49 & 0,23 & 23,93 & 0,22 & 1,86 & 0,19 & 33,59 & 4,00 & 0,33 & 0,04 \\
\hline 16/jan. & 218,59 & 0,92 & 47,80 & 6,85 & 24,50 & 0,05 & 1,70 & 0,38 & $-0,13$ & 34,94 & 0,00 & 0,40 & 220,33 & 0,82 & 54,24 & 0,41 & 24,47 & 0,08 & 1,93 & 0,15 & 37,84 & 3,03 & 0,38 & 0,02 \\
\hline $1 /$ fev. & 213,05 & 0,10 & 41,92 & 6,27 & 24,62 & 0,01 & 1,68 & 0,44 & 0,26 & 37,40 & 0,00 & 0,48 & 212,54 & 0,41 & 48,47 & 0,28 & 24,60 & 0,03 & 1,96 & 0,17 & 40,50 & 2,84 & 0,45 & 0,04 \\
\hline 16/fev. & 213,83 & 0,29 & 45,24 & 6,29 & 24,48 & 0,07 & 1,41 & 0,38 & $-0,61$ & 41,04 & $-0,01$ & 0,59 & 213,34 & 0,20 & 51,27 & 0,26 & 24,47 & 0,06 & 1,63 & 0,16 & 46,55 & 6,12 & 0,54 & 0,04 \\
\hline $1 /$ mar. & 200,32 & 0,20 & 42,33 & 6,59 & 24,17 & 0,03 & 1,43 & 0,39 & $-0,04$ & 31,91 & 0,00 & 0,38 & 200,12 & 0,41 & 48,82 & 0,11 & 24,20 & 0,05 & 1,65 & 0,17 & 33,93 & 2,06 & 0,38 & 0,00 \\
\hline $16 /$ mar. & 206,35 & 0,55 & 35,88 & 5,16 & 23,78 & 0,32 & 1,52 & 0,38 & $-0,11$ & 6,86 & 0,00 & 0,12 & 206,81 & 0,09 & 41,29 & 0,24 & 23,79 & 0,33 & 1,76 & 0,14 & 7,38 & 0,63 & 0,13 & 0,02 \\
\hline 1/abr. & 187,52 & 0,33 & 35,20 & 5,46 & 23,26 & 0,02 & 1,38 & 0,38 & 0,08 & 19,14 & 0,00 & 0,27 & 188,05 & 0,19 & 40,44 & 0,22 & 23,28 & 0,05 & 1,58 & 0,18 & 23,31 & 4,09 & 0,31 & 0,03 \\
\hline 16/abr. & 181,83 & 0,30 & 33,69 & 4,70 & 22,02 & 0,02 & 2,13 & 0,48 & $-0,10$ & 1,26 & 0,00 & 0,05 & 181,28 & 0,26 & 38,32 & 0,07 & 22,02 & 0,02 & 2,46 & 0,14 & $-1,78$ & 2,94 & 0,02 & 0,03 \\
\hline $1 /$ maio & 176,42 & 0,04 & 8,68 & 4,15 & 20,40 & 0,30 & 2,16 & 0,45 & 1, & 5,71 & 0,02 & 0,10 & 176,49 & 0,10 & 32,75 & 0,08 & 20,37 & 0,27 & 2,47 & 0,14 & 7,80 & 0,94 & 0,12 & 0,01 \\
\hline $16 /$ maio & 149,17 & 0,49 & 32,48 & 4,71 & 19,24 & 0,03 & 2,06 & 0,40 & $-0,1$ & 9,32 & 0,00 & 0,12 & 149,71 & 0,05 & 37,01 & 0,18 & 19,24 & 0,04 & 2,36 & 0,10 & 10,97 & 1,84 & 0,12 & 0,00 \\
\hline 1/jun. & 149,06 & 0,32 & 9,07 & 4,23 & 17,32 & 0,10 & 2,60 & 0,39 & $-0,64$ & 11,30 & $-0,01$ & 0,13 & 148,76 & 0,02 & 33,42 & 0,12 & 17,35 & 0,12 & 3,00 & 0,01 & $-18,70$ & 6,76 & $-0,14$ & 0,01 \\
\hline 16/jun. & 145,93 & 0,11 & 24,70 & 3,59 & 17,54 & 0,03 & 2,09 & 0,36 & 0,40 & 12,95 & 0,01 & 0,20 & 57 & 0,25 & 28,39 & 0,10 & 17,59 & 0,01 & 2,40 & 0,06 & 16,62 & 3,27 & 0,24 & 0,02 \\
\hline 1/jul. & 150,07 & 0,08 & 21,63 & 3,20 & 18,11 & 0,01 & 2,05 & 0,37 & 0,53 & 1,14 & 0,02 & 0,06 & 09 & 0,10 & 24,92 & 0,09 & 18,13 & 0,02 & 2,38 & 0,04 & & 3,52 & 0,01 & 0,05 \\
\hline 16/jul. & 153,07 & 0,19 & 26,71 & 4,24 & 17,86 & 0,23 & 2,60 & 0,43 & 0,25 & 1,89 & 0,01 & 0,07 & 153,19 & 0,31 & 31,14 & 0,19 & 17,79 & 0,16 & 2,93 & 0,10 & $-3,21$ & 1,57 & $-0,06$ & 0,00 \\
\hline 1/ago. & 170,75 & 0,10 & 24,25 & 3,35 & 18,00 & 0,13 & 2,62 & 0,46 & 0,28 & 20,55 & 0,01 & 0,34 & 171,09 & 0,23 & 27,58 & 0,02 & 18,00 & 0,13 & 2,93 & 0,14 & 19,24 & 1,59 & 0,32 & 0,03 \\
\hline 16/ago. & 182,69 & 0,01 & 27,13 & 3,94 & 19,01 & 0,23 & 2,75 & 0,45 & 0,28 & 12,27 & 0,00 & 0,16 & 182,58 & 0,13 & 30,88 & 0,19 & 19,04 & 0,20 & 3,16 & 0,05 & $-13,07$ & 1,09 & $-0,16$ & 0,00 \\
\hline 1/out. & 198,80 & 0,21 & 43,10 & 6,44 & 21,11 & 0,12 & 1,98 & 0,41 & $-0,76$ & 10,24 & $-0,01$ & 0,09 & 198,91 & 0,32 & 49,60 & 0,06 & 21,12 & 0,13 & 2,25 & 0,13 & 9,81 & 0,33 & 0,08 & 0,00 \\
\hline 16/out. & 203,91 & 0,08 & 49,76 & 7,20 & 22,50 & 0,10 & 2,38 & 0,46 & 0,79 & 10,17 & 0,00 & 0,01 & 204,72 & 0,72 & 57,14 & 0,18 & 22,49 & 0,09 & 2,73 & 0,11 & $-1,16$ & 12,12 & 0,02 & 0,00 \\
\hline 1/nov & 216,41 & 0,23 & 45,16 & 6,53 & 22,78 & 0,15 & 2,48 & 0,51 & $-0,05$ & 10,48 & 0,00 & 0,06 & 216,78 & 0,14 & 50,72 & 0,97 & 22,79 & 0,16 & 2,86 & 0,14 & 1,94 & 8,49 & 0,04 & 0,02 \\
\hline $16 /$ nov. & 239,33 & 0,37 & 44,52 & 6,29 & 23,09 & 0,07 & 2,19 & 0,46 & $-1,18$ & 18,78 & $-0,01$ & 0,19 & 239,59 & 0,10 & 50,59 & 0,21 & 23,11 & 0,05 & 2,54 & 0,12 & 20,16 & 2,57 & 0,16 & 0,02 \\
\hline 1/dez. & 219,78 & 0,32 & 49,73 & 7,63 & 23,38 & 0,01 & 1,71 & 0,39 & 0,26 & 43,20 & 0,00 & 0,46 & 219,32 & 0,78 & 57,34 & 0,02 & 23,38 & 0,01 & 1,97 & 0,14 & 55,97 & 12,51 & 0,45 & 0,01 \\
\hline 16/dez. & 222,63 & 0,16 & 46,01 & 7,34 & 23,79 & 0,09 & 1,68 & 0,39 & $-0,1$ & 21,60 & 0,00 & 0,27 & 223,59 & 0,80 & 52,91 & 0,44 & 23,80 & 0,07 & 1,92 & 0,15 & 31,04 & 9,56 & 0,25 & 0,02 \\
\hline Média & & 0,26 & & 5,50 & & 0,10 & & 0,42 & & 18,05 & & 0,22 & & 0,28 & & 0,21 & & 0,10 & & 0,12 & & 4,15 & & 0,02 \\
\hline
\end{tabular}

${ }^{(1)}$ Campinas (1960-2004), Cravinhos (1997-2002), Capão Bonito (1991-2002), Gália (1977-2002), Itararé (1977-2000), Jaú (1955-2002), Jundiaí (1993-1999), Limeira (1980-2004), Mococa (1959-2000), Monte Alegre do Sul (1981-2000), Pariqüeraçu (1980-2000), Pindamonhangaba (1982-2000), Pindorama (1965-2000), Piracicaba (1926-2005), Ribeirão Preto (1965-2000), Tatuí (1992-2000) e Tietê (1991-2000). 
e a temperatura do ar era constante. Os autores aplicaram o índice de concordância de Willmott, o coeficiente angular da reta e o índice de desempenho de Camargo, para 24 diferentes datas de semeadura (1으 e $15^{\circ}$ dias de cada mês), para avaliar a eficiência das simulações. Porém, não aplicaram os testes de Bartlett, $\mathrm{F}$ e $\mathrm{t}$, entre os valores paramétricos e os simulados, tampouco verificaram a distribuição de probabilidades correspondentes aos dados observados e simulados. Observaram que apesar de as conclusões expressarem que as simulações apresentam elevados índices de exatidão e precisão, deveriam ainda efetuar mais testes para avaliar melhor os processos de simulação. Mesmo assim, pela metodologia empregada, verificaram que as estimativas de produtividade potencial gerada (Assis et al., 2006) representam a produtividade potencial para a cultura do milho em Piracicaba, SP. Dessa forma, neste trabalho, levando-se em consideração os testes estatísticos utilizados, verificou-se a superioridade na simulação dos dados de radiação fotossinteticamente ativa e temperatura do ar pela simulação normal bivariada.
Além da similaridade entre os parâmetros e os valores simulados, devem-se observar outros critérios para poder usar uma determinada simulação com maior convicção sobre como melhor fazer a simulação de dados. Foram aplicados o teste $\mathrm{F}$ e o de Bartlett, relativos à homogeneidade de variância, entre as variâncias observadas de um determinado dia e as variâncias simuladas desse mesmo dia (Tabela 4). A partir desses resultados, verificaram-se os casos de rejeições a esses testes. Apesar de os valores simulados por meio da distribuição triangular assimétrica e normal truncada serem aparentemente semelhantes, para as duas variáveis, verifica-se que a variância paramétrica e dos dados simulados são heterogêneos, o que indica que houve alteração, no conjunto de dados simulados, que implica na mudança da amplitude da variância, fato que não ocorreu no processo de simulação bivariada.

Verificou-se, ainda, pelo teste $\mathrm{t}$ (comparação das médias paramétricas com as estimativas das médias simuladas para um determinado dia juliano) que em aproximadamente $33 \%$, dos 6.222 casos (17 locais $\mathrm{x}$ 366 dias do ano), a média estimada de $\mathrm{R}$ e $\mathrm{T}$, com os dados simulados pela distribuição triangular assimétrica e normal truncada, diferem dos respectivos parâmetros.

Tabela 3. Estimativa do coeficiente de correlação ( $\rho)$, entre os valores observados e os valores simulados, em 24 datas, por diferentes distribuições (triangular, normal truncada e normal bivariada), para os 17 locais ${ }^{(1)}$ do Estado de São Paulo, nos 375 dias do ano, e respectivos índice de concordância de Willmott (ID), coeficiente angular da reta (b) e índice de desempenho de Camargo (C), para a radiação fotossinteticamente ativa (RFA) e temperatura do ar (Temp).

\begin{tabular}{|c|c|c|c|c|c|c|c|c|c|c|c|c|c|c|c|c|c|c|c|c|c|c|c|c|}
\hline \multirow[t]{3}{*}{ Datas } & \multicolumn{8}{|c|}{ Triangular } & \multicolumn{8}{|c|}{ Normal truncada } & \multicolumn{8}{|c|}{ Normal bivariada } \\
\hline & \multicolumn{4}{|c|}{ RFA } & \multicolumn{4}{|c|}{ Temp } & \multicolumn{4}{|c|}{ RFA } & \multicolumn{4}{|c|}{ Temp } & \multicolumn{4}{|c|}{ RFA } & \multicolumn{4}{|c|}{ Temp } \\
\hline & $\rho$ & ID & b & C & $\rho$ & ID & b & C & $\rho$ & ID & b & C & $\rho$ & ID & b & $\mathrm{C}$ & $\rho$ & ID & b & C & $\rho$ & ID & $\mathrm{b}$ & C \\
\hline /jan. & 0,82 & 0,98 & 1,00 & 00 & 0,64 & 0,96 & 0,98 & 0 & 0,82 & 0,98 & & & 0,64 & 0,96 & 0 & & 0,98 & 0,99 & 1,00 & & 0,99 & 0,99 & 0,99 & 98 \\
\hline & & 98 & & & & & & & 25 & & & & & & & & & & & & & 99 & 00 & \\
\hline & & 98 & & & 51 & & 98 & & 80 & & & & & & & & 98 & & & & & & & \\
\hline & & 98 & & & 68 & & 99 & & 85 & & & & & & & & & & & & & 0,99 & & \\
\hline & 78 & 99 & 97 & 0,9 & 56 & 95 & 86 & & 78 & & & & & 0,95 & 0 , & & & & 1,00 & & 96 & 99 & 00 & \\
\hline & & & & & & & & & & & & & & & & & & & & & & 99 & 99 & 98 \\
\hline & & & & & & & & & & & & & & & & & & & & & & & & \\
\hline & & & & & & & & & & & & & & & & & & & & & & & & \\
\hline & & & & & & & & & & & & & & & & & & & 00 & & & 0,99 & 00 & \\
\hline & & 99 & & 0 &, 66 & &, 97 & & & & & & & & & & & & 00 & & & 99 & 00 & \\
\hline & & & & & & & & & & & & & & & & & & & & & & & & \\
\hline & & & & & & & & & & & & & & & & & & & & & & & & \\
\hline & & & & & & & & & & & & & & & & & & & & & & 99 & 0 & \\
\hline & 88 & 99 & & & & & 96 & & & & & & & 99 & & & 99 & 99 & 1,00 & & 98 & 0,99 &, 00 & (9), \\
\hline & & & & & & & & & & & & & & & & & & & & & & 9 & 00 & \\
\hline & & & & & & & & & & & & & & & & & & & & & & & & \\
\hline & & & & & & & & & & & & & & & & & & & & & & 9 & 10 & \\
\hline & & & & & & & & & & & & & & & & & & & 00 & & & 0,99 & 00 & \\
\hline & 36 & & & & & & & & & & & & & & & & & & 1,00 & & 97 & 0,99 & ,00 & 99 \\
\hline & & & & & & & & & & & & & & & & & & & & & & & 00 & \\
\hline & & & & & & & & & & & & & & & & & & & & & & & & \\
\hline & & & & & & & & & & & & & & & & & & & & & & & & \\
\hline & & & & & & & & & & & & & & & & & & & & & & 0,99 & 1,00 & ( \\
\hline 6/dez. & 0,86 & 0,99 & 00 & 0,98 & 0,44 & 0,97 & 0,84 & 0,81 & 0,86 & 0,99 & 1,00 & 0,98 & 0,44 & 0,97 & 0,84 & 0,81 & 0,98 & 0,99 & 1,00 & 0,99 & 0,97 & 0,99 & 1,00 & 0,99 \\
\hline
\end{tabular}

${ }^{(1)}$ Campinas (1960-2004), Cravinhos (1997-2002), Capão Bonito (1991-2002), Gália (1977-2002), Itararé (1977-2000), Jaú (1955-2002), Jundiaí (1993-1999), Limeira (1980-2004), Mococa (1959-2000), Monte Alegre do Sul (1981-2000), Pariqüeraçu (1980-2000), Pindamonhangaba (1982-2000), Pindorama (1965-2000), Piracicaba (1926-2005), Ribeirão Preto (1965-2000), Tatuí (1992-2000) e Tietê (1991-2000). 
Em contrapartida, a simulação dos dados de R e T, pela distribuição normal bivariada, indicou que as estimativas da média e desvio-padrão não divergem dos respectivos parâmetros, para todos os locais e em geral. Além disso, as diferenças (DIF) entre as correlações ( $\mathrm{R}$ e T) paramétricas e estimadas com os dados simulados são muito pequenas (Tabela 2).

Assis et al. (2006) utilizaram a distribuição triangular para simular os valores de radiação solar e temperatura

Tabela 4. Número de casos em que os 1.000 valores de radiação fotossinteticamente ativa (RFA) e temperatura do ar (Temp) simulados pelas distribuições triangular assimétrica (Tri), normal truncada (NT) e normal bivariada (NB), em cada um dos 17 locais $^{(1)}$ no Estado de São Paulo, nos 365 dias do ano, não foi normal pelo teste Kolmogorov-Smirnov, número de casos em que as hipóteses dos testes de Bartlett, t e F foram rejeitadas.

\begin{tabular}{|c|c|c|c|c|c|c|}
\hline \multirow[t]{2}{*}{ Locais } & \multicolumn{3}{|c|}{ RFA } & \multicolumn{3}{|c|}{ Temp } \\
\hline & Tri & NT & $\mathrm{NB}$ & Tri & NT & NB \\
\hline Campinas & 307 & 2 & 0 & 163 & 4 & 0 \\
\hline Cravinhos & 269 & 4 & 0 & 236 & 5 & 0 \\
\hline Capão Bonito & 234 & 3 & 0 & 257 & 7 & 0 \\
\hline Gália & 271 & 2 & 0 & 169 & 3 & 0 \\
\hline Itararé & 225 & 2 & 0 & 134 & 4 & 0 \\
\hline Jaú & 327 & 3 & 0 & 136 & 3 & 0 \\
\hline Jundiaí & 269 & 4 & 0 & 169 & 4 & 0 \\
\hline Limeira & 155 & 6 & 0 & 115 & 2 & 0 \\
\hline Mococa & 291 & 6 & 0 & 148 & 2 & 0 \\
\hline Monte Alegre do Sul & 309 & 4 & 0 & 116 & 2 & 0 \\
\hline Pariquera/Açú & 196 & 4 & 0 & 104 & 5 & 0 \\
\hline Pindamonhangaba & 295 & 4 & 0 & 164 & 3 & 0 \\
\hline Pindorama & 294 & 2 & 0 & 154 & 2 & 0 \\
\hline Piracicaba & 292 & 3 & 0 & 134 & 2 & 0 \\
\hline Ribeirão Preto & 303 & 4 & 0 & 281 & 4 & 0 \\
\hline Tatuí & 259 & 3 & 0 & 140 & 5 & 0 \\
\hline Tietê & 271 & 4 & 0 & 215 & 5 & 0 \\
\hline Total & 4.567 & 60 & 0 & 2.835 & 62 & 0 \\
\hline Porcentual total & 73,40 & 1,0 & 0 & 45,60 & 1,0 & 0 \\
\hline Bartlett & 5.636 & 64 & 0 & 2.370 & 307 & 0 \\
\hline $\mathrm{t}$ & 2.052 & 0 & 0 & 2.104 & 0 & 1 \\
\hline $\mathrm{F}$ & 5.902 & 335 & 0 & 3.144 & 834 & 0 \\
\hline
\end{tabular}

(1)Campinas (1960-2004), Cravinhos (1997-2002), Capão Bonito (1991-2002), Gália (1977-2002), Itararé (1977-2000), Jaú (1955-2002), Jundiaí (1993-1999), Limeira (1980-2004), Mococa (1959-2000), Monte Alegre do Sul (1981-2000), Pariqüeraçu (1980-2000), Pindamonhangaba (1982-2000), Pindorama (1965-2000), Piracicaba (1926-2005), Ribeirão Preto (1965-2000), Tatuí (1992-2000) e Tietê (1991-2000). diária, e usaram esses dados no modelo para o cálculo do rendimento potencial. $\mathrm{Na}$ avaliação do processo de simulação, também utilizaram o índice de concordância de Willmott, coeficiente angular da reta e índice de desempenho de Camargo, para 24 diferentes datas de semeadura (1o e 15o dias de cada mês). Porém, não foram aplicados os testes de Bartlett, F e t, entre os valores paramétricos e simulados, tampouco foi verificada a distribuição de probabilidades correspondentes aos dados observados e simulados. No estudo supracitado, os valores do coeficiente de correlação entre $\mathrm{R}$ e $\mathrm{T}$ variaram, aproximadamente, de 0,50 a 0,95 . O índice de concordância de Wilmott apresentou valores de variação semelhantes, e o coeficiente angular da regressão simples apresentou valores superiores a 0,94 . Ao se considerarem somente os valores apresentados por esses critérios de avaliação, para um tipo de simulação, não se faz difícil dar confiabilidade a determinado tipo de simulação de dados. Porém, no caso da simulação de processos que são correlacionados, presentes na natureza, devem-se comparar mais métodos de simulação, além de se aplicar maior número de testes para a melhor decisão. Certamente, a simulação triangular se adapta aos casos em que é possível se determinar o valor mais provável da variável aleatória, os seus valores mínimo e máximo e aos casos em que uma função linear parece apropriada, para a descrição da distribuição dos valores dos erros das variáveis.

As variáveis simuladas devem apresentar um comportamento similar, quando comparadas com as variáveis que originam os parâmetros para sua geração. Pelos resultados mostrados anteriormente, observa-se que a grande maioria dos dias do ano apresenta dados que aderem à distribuição normal de probabilidade. Então, isso também deve ocorrer para as variáveis simuladas. O teste de normalidade aplicado sobre o conjunto de 1.000 valores simulados, a partir de cada um dos processos de simulação realizados, pode identificar para cada variável (R e T) o número de nãoaderências (ou rejeições) que os dados simulados apresentam. O número de rejeições à distribuição de probabilidade normal, separado para cada uma das variáveis ( $\mathrm{R} \mathrm{e} \mathrm{T),} \mathrm{é} \mathrm{apresentado} \mathrm{na} \mathrm{Tabela} \mathrm{4,} \mathrm{para} \mathrm{cada}$ um dos locais, em cada processo de geração dos valores simulados. Por esses resultados, verifica-se que os valores simulados pela distribuição triangular apresentam rejeição à distribuição normal de probabilidade de $73,40 \%$, para a variável radiação fotossinteticamente ativa, e de 
45,60\% para a temperatura média diária. A simulação pela distribuição normal a $\pm 1,96$ desvio-padrão, praticamente não apresentou rejeições à distribuição normal, tendo resultado em aproximadamente $1 \%$ dos casos, para ambas as variáveis. Em contrapartida, a simulação dos dados, pela distribuição normal bivariada, não apresentou nenhuma rejeição da normalidade aos dados simulados.

Hair et al. (2005) indicam que se a normalidade no sentido univariado não é violada, isso nem sempre resultará em que normalidade multivariada seja respeitada, mas certamente ajudará na obtenção da normalidade multivariada. Porém, a normalidade multivariada significa que as variáveis individuais são normais em um sentido univariado, e que suas combinações também são normais. Logo, se uma variável é normal multivariada, também é normal univariada. Então, a simulação dos dados, pela distribuição normal bivariada, produz dados mais favoráveis para que a normalidade multivariada não seja rejeitada. No caso dos valores simulados por meio das distribuições triangular assimétrica e normal truncada, essas certamente resultarão em um maior número de rejeições à distribuição de probabilidade normal multivariada. Santos \& Ferreira (2003a) determinaram o tamanho amostral para as estatísticas univariadas $\left(\mathrm{Z}_{1}\right.$ e $Z_{2}$ ) e multivariadas $\left(K_{1}\right.$ e $\left.K_{2}\right)$, tendo considerado as medidas de assimetria $\left(Z_{1}\right.$ e $\left.K_{1}\right)$ e de curtose $\left(Z_{2}\right.$ e $\left.K_{2}\right)$. Os autores verificaram que as estatísticas $Z_{1}$ e $Z_{2}$ possuem aproximações assintóticas normais, para $\mathrm{n} \geq 25$ e $\alpha=5 \%$, e podem ser recomendadas para uso rotineiro no caso univariado, para testar a hipótese de normalidade dos dados. Além disso, as estatísticas $\mathrm{K}_{1}$ e $\mathrm{K}_{2}$ possuem aproximações assintóticas melhores que $\mathrm{Z}_{1}$ e $\mathrm{Z}_{2}$, para menor valor de significância, e são recomendadas para $\mathrm{n} \geq 25$ e $\mathrm{n} \geq 100$, respectivamente, as quais mantêm o controle da taxa de erro tipo I e elevado poder (superior a $80 \%$ ). Além disso, no caso de distribuições com simetria próxima de zero e não normais, as estatísticas baseadas em desvios de simetria apresentam maior poder do que a estatística W de Shapiro \& Wilk (1965).

A verificação da normalidade multivariada é realizada pela verificação da normalidade da distribuição marginal univariada (Bock, 1975). Sabe-se que a existência de uma distribuição normal multivariada implica em marginais normalmente distribuídas, porém, não se pode garantir que a distribuição conjunta das duas variáveis normais univariadas sejam uma normal multivariada (Santos \& Ferreira, 2003b).

\section{Conclusão}

A simulação de dados de radiação fotossinteticamente ativa e temperatura do ar é melhor realizada pela distribuição normal bivariada, em comparação com as simulações pela distribuição triangular e a distribuição normal truncada.

\section{Agradecimentos}

Ao CNPq, pela concessão de bolsa; ao IAC e ao Departamento de Ciências Exatas, da Esalq, pela disponibilização dos dados.

\section{Referências}

ALLEN, D.J.; ORT, D.R. Impacts of chilling temperatures on photosynthesis in warm-climate plants. Trends in Plant Science, v.6, p.36-42, 2001.

ASSIS, J.P.; DOURADO NETO, D.; NASS, L.L.; MANFRON, P.A.; BONNECARRÈRE, R.A.G.; MARTIN, T.N. Simulação estocástica de atributos do clima e da produtividade potencial de milho utilizando-se distribuição triangular. Pesquisa Agropecuária Brasileira, v.41, p.539-543, 2006.

BOCK, R.D. Multivariate statistical methods in behavior research. Chicago: MacGraw-Hill, 1975. 623p.

BOX, G.E.P.; MULLER, M.E. A note on the generation of random normal derivates. The Annals of Mathematical Statistics, v.29, p.610-611, 1958.

CAMARGO, A.P.; SENTELHAS, P.C. Avaliação do desempenho de diferentes métodos de estimativa da evapotranspiração potencial no Estado de São Paulo, Brasil. Revista Brasileira de Agrometeorologia, v.5, p.89-97, 1997.

CAMPOS, H. Estatística experimental não paramétrica. 4.ed. Piracicaba: Esalq-USP, 1983.349p.

CARVALHO JUNIOR, W.; CHAGAS, C.S.; PEREIRA, N.R.; STRAUCH, J.C.M. Elaboração de zoneamentos agropedoclimáticos por geoprocessamento: soja em municípios do Rio Grande do Sul. Revista Brasileira de Ciência do Solo, v.27, p.379-387, 2003.

DETOMINI, E.R.; DOURADO NETO, D. Variação temporal da fitomassa seca relativa de Brachiaria brizantha cultivares Marandu e Xaraés. Pasturas Tropicales, v.27, p.13-21, 2005.

DOURADO NETO, D.; ASSIS, J.P.; TIMM, L.C.; MANFRON, P.A.; SPAROVEK, G.; MARTIN, T.N. Ajuste de modelos de distribuição de probabilidade a séries históricas de precipitação pluvial diária em Piracicaba-SP. Revista Brasileira de Agrometeorologia, v.13, p.273-283, 2005.

DOURADO NETO, D.; FIGUEREDO JUNIOR, L.G.M.; NOVA, N.A.V.; LIMA, M.G.; MANFRON, P.A.; MEDEIROS, S.L.P. Modelos para estimação de assimilação de dióxido de carbono, coeficiente de extinção de radiação solar e produtividade de grãos da cultura de milho. Revista Brasileira de Agrometeorologia, v.12, p.349-353, 2004. 
GENNEVILLE, M.S.; BOOCK, A. Modelo estocástico para simulação da precipitação pluviométrica diária de uma região. Pesquisa Agropecuária Brasileira, v.18, p.959-966, 1983.

HAIR, J.F.; ANDERSON, R.E.; TATHAM, R.L.; BLACK, W.C. Análise multivariada de dados. 5.ed. Porto Alegre: Bookman, 2005. 595p.

HOGG, R.V.; CRAIG, A.T. Introduction to mathematical statistics. $4^{\text {th }}$ ed. New York: Macmillan, 1978. 438p.

KORTUM, S. Lecture 4: selection. Minneapolis: University of Minnesota; Department of Economics, 2002. 3p. Disponível em: www.econ.umn.edu/ kortum/courses/fall02/lecture4k.pdf. Acesso em: 15 dez. 2003.

MAJOR, D.J.; JOHNSON, D.R.; LUEDDERS, V.D. Evaluation of eleven thermal unit methods for predicting soybean development. Crop Science, v.15, p.172-174, 1975.

MORETTIN, P.A.; BUSSAB, W.O. Estatística básica. 5.ed. São Paulo: Saraiva, 2003. 526p.

PEREIRA, A.R.; ANGELOCCI, L.R.; SENTELHAS, P.C. Agrometeorologia: fundamentos e aplicações práticas. Guaíba: Ed. Agropecuária, 2002. 478p.

PESSOA, M.C.P.Y.; LUCHIARI JUNIOR, A.; FERNANDES, E.N.; LIMA, M.A. de. Principais modelos matemáticos e simuladores utilizados para a análise de impactos ambientais das atividades agrícolas. Jaguariúna: Embrapa-CNPMA, 1997. 83p. (EmbrapaCNPMA. Documentos, 8).

SANTOS, A.C.; FERREIRA, D.F. Definição do tamanho amostral usando simulação Monte Carlo para o teste de normalidade baseado em assimetria e curtose. I. Abordagem univariada. Ciência e Agrotecnologia, v.27, p.432-437, 2003a.

SANTOS, A.C.; FERREIRA, D.F. Definição do tamanho amostral usando simulação Monte Carlo para o teste de normalidade baseado em assimetria e curtose. II. Abordagem multivariada. Ciência e Agrotecnologia, v.27, p.62-69, 2003b.

SHAPIRO, S.S.; WILK, M.B. An analysis of variance test for normality. Biometrika, v.52, p.591-611, 1965.

SONOIKE, K. Various aspects of inhibition of photosynthesis under light/chilling stress: photoinhibition at chilling temperatures versus chilling damage in the light. Journal of Plant Research, v.111, p.121-129, 1998.

SOUSA, S.A.V. Programa computacional para simulação da ocorrência de veranicos e queda de produção. 1999. 124p. Tese (Doutorado) - Universidade de São Paulo, Piracicaba.

STEEL, R.G.D.; TORRIE, J.H.; DICKEY, D.A. Principles and procedures of statistics: a biometrical approach. 3.ed. New York: McGraw Hill Book, 1997. 666p.

VAN HEEMST, H.D.J. Physiological principles. In: VAN KEULEN, H.; WOLF, J. Modeling of agricultural production: weather, soils and crops. Wageningem: Pudoc, 1986. p.13-26.

VILLA NOVA, N.A.; PEDRO JÚNIOR, M.J.; PEREIRA, A.R.; OMETTO, J.C. Estimativa de graus-dia acumulado acima de qualquer temperatura base, em função das temperaturas máxima e mínima. Caderno de Ciências da Terra, v.30, p.1-7, 1972.

WILlmotT, C.J. On the validation of models. Physical Geography, v.2, p.184-194, 1981.

Recebido em 22 de janeiro de 2007 e aprovado em 1o de agosto de 2007 